\title{
The effect of e-cigarettes on smoking cessation and cigarette smoking initiation: An evidence-based rapid review and meta-analysis
}

\author{
Ying-Ying Zhang', Fan-Long Bu 1,2, Fei Dong', Jian-Hua Wang ${ }^{3}$, Si-Jia Zhu' , Xiao-Wen Zhang', Nicola Robinson ${ }^{1,4}$, Jian-Ping Liu'
}

\begin{abstract}
INTRODUCTION The contribution made by e-cigarettes to smoking cessation continues to be controversial. Reports suggest that teenagers are becoming increasingly addicted to e-cigarettes and that e-cigarette use in adolescents is associated with subsequent cigarette smoking.

METHODS Systematic searches of eleven databases were conducted (January 2015 to June 2020). Systematic reviews, randomized controlled trials (RCTs) and cohort studies comparing e-cigarettes with placebo e-cigarettes, nicotine replacement therapy (NRT) or no e-cigarette use were included. The two primary outcomes were smoking cessation among smokers and smoking initiation among non-smoking teenagers. The secondary outcome was adverse events. Data were synthesized using risk ratio (RR) or adjusted odds ratio (AOR) with 95\% confidence interval (CI).

RESULTS Six systematic reviews, 5 RCTs and 24 cohort studies were identified. For smoking cessation, findings from 4 systematic reviews indicated that e-cigarettes contributed to cessation while one found the opposite. Meta-analysis of 5 RCTs suggested that e-cigarettes were superior to NRT or placebo for smoking cessation $\left(\mathrm{RR}=1.55 ; 95 \%\right.$ CI: $1.00-2.40 ; \mathrm{I}^{2}=57.6 \%$; low certainty; 5 trials, $\left.\mathrm{n}=4025\right)$. Evidence from 9 cohort studies showed that e-cigarette use was not associated with cessation (AOR=1.16; 95\% CI: 0.88-1.54; $\mathrm{I}^{2}=69.0 \%$; $\left.=22220\right)$. Subgroup analysis suggested that intensive e-cigarette use may be associated with cessation. In terms of smoking initiation, adolescents who ever used e-cigarettes had a greater risk for smoking initiation than non-users ( $\mathrm{AOR}=2.91 ; 95 \%$ CI: $2.61-3.23 ; \mathrm{I}^{2}=61.0 \%$; 15 trials, $n=68943$ ), the findings were consistent with one included systematic review. No serious adverse events were reported in the included studies.

CONCLUSIONS Low certainty evidence suggests that e-cigarettes appear to be potentially effective for smoking cessation. The use of e-cigarettes in adolescents may be associated with smoking initiation. No serious adverse events were reported.
\end{abstract}

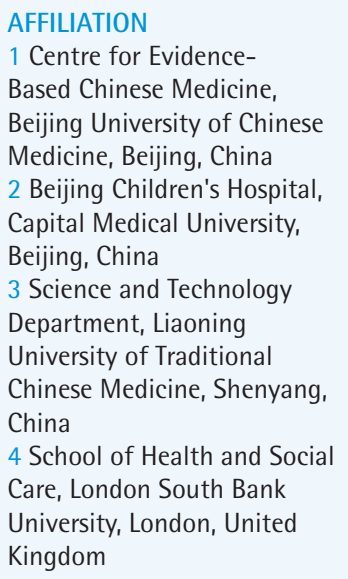

CORRESPONDENCE TO Jian-Ping Liu. Centre for Evidence-Based Chinese Medicine, Beijing University of Chinese Medicine, $11 \mathrm{Bei}$ San Huan Dong Lu, Chaoyang District, Beijing 100029, China. E-mail: liujp@bucm.edu.cn

\section{KEYWORDS}

e-cigarettes, smoking cessation, smoking initiation rapid review, systematic review

Received: 30 August 2020 Revised: 22 October 2020

Accepted: 12 December 2020

\section{INTRODUCTION}

Electronic cigarettes, also called e-cigarettes are electronic nicotine delivery systems (ENDS). They can deliver vaporized liquid mainly containing nicotine and propylene glycol through the mouth into the lungs, mimicking the effects of conventional cigarette smoking ${ }^{1}$. Reportedly, the e-cigarette was invented by the Chinese pharmacist Hon Lik in 2003, and internationally patented in $2007^{2}$. E-cigarettes have been commercially available in Europe and America since 2006 as a cigarette substitute ${ }^{3}$. E-cigarettes have always been advertised as healthy and popular 
smoking cessation tools ${ }^{4}$. Reports have suggested that achieving abstinence by using e-cigarettes was comparable to nicotine replacement therapy (NRT), and e-cigarettes also appear to effectively decrease cigarette consumption ${ }^{5,6}$. This has resulted in a dramatic increase in the use of e-cigarettes worldwide. The estimated global value of e-cigarette sales was up to US $\$ 3.5$ billion in $2015^{7}$. A national large-scale survey from $28 \mathrm{EU}$ member states reported that there were about 48.5 million e-cigarette users in $2016^{8}$.

Increasingly teenagers are becoming addicted to e-cigarettes. Between 2016 and 2017, the proportion of e-cigarette users among those aged 11 to 16 years increased from $7 \%$ to $11 \%$, respectively, across the $\mathrm{UK}^{9}$. The proportion of US high school teenagers who had tried e-cigarettes in the last month rose from $1.5 \%$ to $16.0 \%$ between 2011 and 2015 , a more than 10 -fold increase ${ }^{10}$. In 2019 , it was estimated that among high school students in the US, $27.5 \%$ (95\% CI: 25.3-29.7) were current e-cigarette users ${ }^{11}$. Worse still, many studies appear to report that use of e-cigarettes by young people increases the risk of subsequent cigarette smoking ${ }^{12}$, and that minors are an emerging new smoking population ${ }^{13}$.

Concerns have been expressed about the safety of e-cigarettes. The US Food and Drug Administration (FDA) reported that e-cigarettes contained some toxic heavy metals and the concentration of heavy metals released by e-cigarettes was much higher than conventional cigarettes ${ }^{14}$. These were associated with the development of cancer ${ }^{15}$ and the occurrence of coronary events ${ }^{16}$. In light of the above, the FDA banned flavored e-cigarette sales in January 2020, as flavored e-cigarettes had the most appeal to teenagers ${ }^{17}$. On 1 November 2019 , China as the birthplace of e-cigarettes announced that the online purchase of e-cigarettes would be completely banned in China ${ }^{18}$. Previously, two randomized controlled trials ( RCTs) ${ }^{5,6}$ indicated that e-cigarettes were potentially effective in smoking cessation among adult smokers. Published studies on cigarette smoking initiation associated with e-cigarette use among adolescents have been primary studies and there has been limited evidence synthesis. There are few evidence-based studies that have comprehensively evaluated the benefits and risks of e-cigarettes. Therefore, a rapid review was conducted to evaluate the effects of e-cigarettes on smoking cessation among smokers and the risks for smoking initiation among non-smoking adolescents, and their safety.

\section{METHODS}

Due to the urgent need to inform ongoing policy, a rapid review methodology was employed ${ }^{19}$. This type of simplified systematic review is helpful in providing a timely synthesis for decision makers. Streamlined methods are usually used in a rapid review, which usually includes limiting retrieval dates and database ${ }^{19}$. This rapid review and meta-analysis are reported following Preferred Reporting Items for Systematic Reviews and Meta-Analyses (PRISMA) ${ }^{20}$ statement. A PRISMA statement with a checklist of items that should be included in reports of systematic reviews is included in the Supplementary file.

\section{Eligibility criteria}

Systematic reviews based on RCTs or comparative observational studies (cohort and cross-sectional studies), parallel group RCTs and controlled cohort studies were included. The study population comprised adult smokers and non-smoking adolescents, who had no serious diseases or pregnancy. The study interventions were e-cigarettes. The controls referred to placebo e-cigarettes (without nicotine), NRT or no treatment. The primary outcomes were smoking cessation among smokers and smoking initiation among adolescents. The secondary outcome was occurrence of adverse events. Experimental studies and studies that failed to report the minimal required information were excluded. There was no need for approval from an ethics committee or agreement of participants in this study as data were extracted from publications that were in the public domain.

\section{Search strategy}

Databases searched included PubMed, Web of Science, MEDLINE, EMBASE, the Cochrane library, China National Knowledge Infrastructure (CNKI), Chinese Scientific Journal Database (VIP), SinoMed and Wanfang, from January 2015 to June 2020. The reference lists included in the systematic reviews were also searched. A systematic search was also conducted in Clinical Trials.gov (www.clinicaltrials.gov) and Chinese Clinical Trial Registry (http://www.chictr.org. cn/index.aspx). Search terms included: e-cigarettes, 
electronic cigarettes, electronic nicotine delivery systems, vape, and vaping. An example of the PubMed search strategy is given in the Supplementary file.

\section{Study selection and data extraction}

After removing duplicates, two authors (SJZ and FLB) independently screened studies by title and abstract, according to the eligibility criteria. In the full text screening process, uncertainty and insufficient information was determined for eligibility through full texts. Disagreements were resolved by discussion or arbitrated by a methodologist (JPL). Reasons for excluding studies were recorded at this stage. After identification of studies, data were extracted independently by two authors (SJZ and FLB) and included: characteristics of study design (study type, setting, and funding); details of PICO (participants, interventions, comparisons, and outcomes); and follow-up and adjusted factors. Any disagreements were resolved by discussion or thirdparty adjudication.

\section{Quality assessments}

The methodological quality assessment of each included study was performed independently by two review authors (FLB and SJZ). Cochrane Risk of Bias tool (ROB $)^{21}$ was employed to assess whether the risk of bias of RCTs was low, high or unclear according to its seven domains. A Measurement Tool to Assess Systematic Reviews (AMSTAR-2) ${ }^{22}$ which has 16 items with 7 key items was employed to assess the quality of the included systematic reviews; the overall confidence for each review was assessed as high, moderate, low or critically low. The quality of cohort studies was assessed by NewcastleOttawa Scale (NOS) ${ }^{23}$, which is composed of 8 items and includes three subscales: selection of studies, comparability of studies, and the ascertainment of exposure. The maximum score is 9 points and any study scoring $>5$ points was considered of moderate to high quality ${ }^{23}$. Grading of Recommendations Assessment, Development and Evaluation (GRADE) ${ }^{24}$ was employed to assess the certainty of evidence from RCTs in five domains (risk of bias, directness, precision, consistencies, and publication bias).

\section{Data synthesis}

Qualitative and quantitative methods were adopted to synthesize the findings. The findings from systematic reviews were narratively described. Data are presented as risk ratios (RRs) or adjusted odds ratio (AORs) with 95\% confidence intervals (CIs). Meta-analysis was performed by Stata version 14.0 software when the trials had acceptable heterogeneity and similarities in clinical characteristics. The random effects model (REM) was utilized in the meta-analysis to consider potential sources of clinical heterogeneity. The $\mathrm{I}^{2}$ statistic was employed to assess statistical heterogeneity ${ }^{21}$. When $\mathrm{p}<0.10$ and $\mathrm{I}^{2}>50 \%$, the heterogeneity may be considered as high ${ }^{21,25}$. To explain heterogeneity, we predefined the subgroup analysis by the frequency of e-cigarette use: intensive use (daily or regular use for at least one month) versus intermittent or irregular use. Sensitivity analysis was employed to test the robustness of the results when the primary outcomes were statistically different for the following three methodological domains: reported clear randomization concealment or not; placebo used or not; and reported lost to follow-up or not. Funnel plots were employed to detect the possibility of publication bias if $\geq 10$ studies were included in a meta-analysis.

\section{RESULTS}

\section{Screening}

Initially, 1620 records were retrieved and 926 duplicates were removed. In all, 612 records were excluded by scanning the title and abstract due to irrelevant studies, protocols, clinical studies, casecontrol studies or cross-sectional surveys, commentary of included studies, and studies that provided only abstracts. This left 82 remaining records, of these, 47 studies were excluded through full-text screening due to ineligible study design, uncontrolled cohort studies or lack of cessation outcomes. Finally, 35 studies were included after full-text screening. Of these studies, findings from 6 systematic reviews ${ }^{26-31}$ were narratively described. The screening process is shown in Figure 1.

\section{Description of studies}

The characteristics of the included studies are shown in Tables 1 and 2 . Included in the 35 studies identified were: 6 systematic reviews ${ }^{26-31}, 5 \mathrm{RCTs}^{32-36}$, and 24 cohort studies $^{37-60}$. These studies were classified into two categories based on the two primary outcome 
Figure 1. Flow chart of study selection

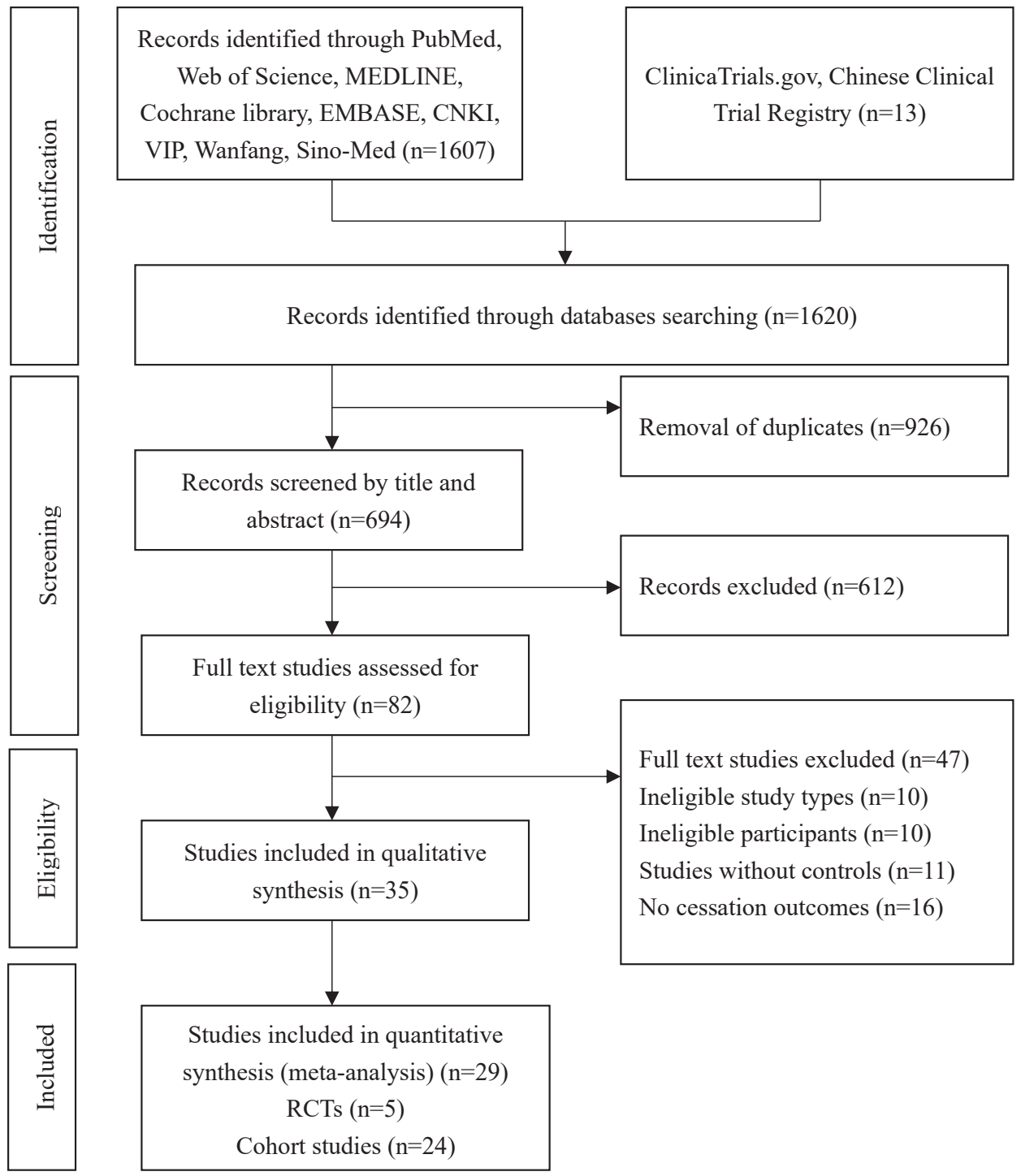

RCTs: randomized controlled trials.

measures. Nineteen studies ${ }^{26-30,32-45}$ examined the effects of e-cigarette use on smoking cessation, and the remaining 16 studies $^{31,46-60}$ explored the causality between e-cigarette use and smoking initiation among teenagers. The study population included adult smokers and non-smoking adolescents and young adults. The definition of e-cigarette use was 'current use', 'ever use' or 'past 30-day use'. In terms of the included 6 systematic reviews, 5 reviews ${ }^{26-30}$ focused on smoking cessation among adult smokers, and included either RCTs, cohort studies or cross-sectional studies. Another systematic review ${ }^{31}$ was based on 9 cohort studies and explored the association between e-cigarette use and subsequent smoking initiation. The $5 \mathrm{RCTs}^{32-36}$ involved data from 4025 adult smokers. The sample size ranged from 99 to 886 . Three trials ${ }^{32,34,35}$ compared the effect of e-cigarettes with NRT on smoking cessation. The other 2 RCTs $^{33,36}$ compared the effect of nicotine e-cigarettes with placebo or no treatment. A total of 24 cohort studies were identified. The sample size ranged from 146 to 17318 with a total of 91985 participants. Nine studies ${ }^{37-45}$ focused on smoking cessation, of which, 4 studies $^{37-39,42}$ overlapped with the included 
Table 1. Characteristics of the included systematic reviews and randomized controlled trials

\begin{tabular}{|c|c|c|c|c|c|}
\hline Study and Year & Location & Population/intervention & Comparison & Outcomes & Included study types \\
\hline $\begin{array}{l}\text { Kalkhoran et al. }{ }^{26} \\
(2016)\end{array}$ & USA & $\begin{array}{l}\text { Adult smokers with nicotine } \\
\text { EC use }\end{array}$ & Non-EC users & $\begin{array}{l}\text { Probability of smoking } \\
\text { cessation }\end{array}$ & $\begin{array}{l}15 \text { cohort studies, } 3 \\
\text { cross-sectional studies } \\
\text { and } 2 \text { clinical trials }\end{array}$ \\
\hline $\begin{array}{l}\text { El Dib et al. }{ }^{27} \\
(2017)\end{array}$ & Brazil & $\begin{array}{l}\text { Adult smokers with nicotine } \\
\text { EC use }\end{array}$ & Placebo EC & $\begin{array}{l}\text { Probability of smoking } \\
\text { cessation }\end{array}$ & $\begin{array}{l}3 \text { RCTs and } 9 \text { prospective } \\
\text { cohort studies }\end{array}$ \\
\hline $\begin{array}{l}\text { Hartmann-Boyce } \\
\text { et al. }{ }^{28}(2016)\end{array}$ & UK & $\begin{array}{l}\text { Adult smokers with nicotine } \\
\text { EC use }\end{array}$ & Placebo EC & $\begin{array}{l}\text { Probability of smoking } \\
\text { cessation }\end{array}$ & $\begin{array}{l}3 \text { RCTs and } 21 \text { cohort } \\
\text { studies }\end{array}$ \\
\hline 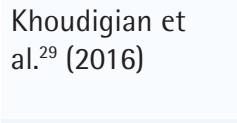 & Canada & $\begin{array}{l}\text { Adult smokers with nicotine } \\
\text { EC use }\end{array}$ & Placebo EC & $\begin{array}{l}\text { Probability of smoking } \\
\text { cessation }\end{array}$ & $\begin{array}{l}3 \text { RCTs and } 2 \\
\text { comparative } \\
\text { observational studies }\end{array}$ \\
\hline $\begin{array}{l}\text { Rahman et al. }{ }^{30} \\
(2015)\end{array}$ & Australia & $\begin{array}{l}\text { Adult smokers with nicotine } \\
\text { EC use }\end{array}$ & Placebo EC & $\begin{array}{l}\text { Probability of smoking } \\
\text { cessation }\end{array}$ & $\begin{array}{l}2 \text { RCTs, } 2 \text { cohort } \\
\text { studies and } 2 \text { cross- } \\
\text { sectional studies }\end{array}$ \\
\hline $\begin{array}{l}\text { Soneji et al. }{ }^{31} \\
(2017)\end{array}$ & Lebanon & $\begin{array}{l}\text { Non-smoking teenagers with } \\
\text { current or past } 30 \text {-day nicotine } \\
\text { EC use }\end{array}$ & $\begin{array}{l}\text { Never or non-past } \\
\text { 30-day EC users }\end{array}$ & $\begin{array}{l}\text { Probability of cigarette } \\
\text { smoking initiation }\end{array}$ & 9 cohort studies \\
\hline $\begin{array}{l}\text { Hajek et al. }{ }^{32} \\
(2019)\end{array}$ & UK & Nicotine EC users $(n=438)$ & NRT $(n=446)$ & 1-year abstinence rate & RCT \\
\hline $\begin{array}{l}\text { Tseng et al. }{ }^{33} \\
\text { (2016) }\end{array}$ & USA & $4.5 \%$ nicotine EC users $(n=50)$ & Placebo EC $(n=49)$ & Abstinence rate at week 3 & $\mathrm{RCT}$ \\
\hline Lee et al..$^{34}$ (2019) & Korea & Nicotine EC users $(n=75)$ & Nicotine gum $(n=75)$ & $\begin{array}{l}\text { Abstinence rate at 9-24 } \\
\text { weeks }\end{array}$ & $\mathrm{RCT}$ \\
\hline Li et al. ${ }^{35}$ (2019) & UK & Nicotine EC users $(n=439)$ & NRT $(n=447)$ & 1-year abstinence rate & RCT \\
\hline $\begin{array}{l}\text { Halpern et al. }{ }^{36} \\
(2018)\end{array}$ & USA & Nicotine EC users $(n=1199)$ & $\begin{array}{l}\text { No treatment } \\
(n=813)\end{array}$ & 6-month abstinence rate & $\mathrm{RCT}$ \\
\hline
\end{tabular}

EC: electronic cigarette. NRT: nicotine replacement therapy.

Table 2. Characteristics of the included cohort studies

\begin{tabular}{|c|c|c|c|c|c|c|c|c|}
\hline $\begin{array}{l}\text { Study and } \\
\text { Year }\end{array}$ & Location & Exposure & Population & Comparison & $\begin{array}{l}\text { Follow- } \\
\text { up }\end{array}$ & Outcomes & Adjusted factors & $\begin{array}{l}\text { Lost to } \\
\text { follow-up } \\
(\%)\end{array}$ \\
\hline $\begin{array}{l}\text { Shi et al. }{ }^{37} \\
(2016)\end{array}$ & USA & $\begin{array}{l}\text { Ever nicotine EC } \\
\text { users }(n=82)\end{array}$ & $\begin{array}{l}2454 \text { current adult } \\
\text { smokers }\end{array}$ & $\begin{array}{l}\text { Non-EC users } \\
(n=936)\end{array}$ & 1 year & $\begin{array}{l}\text { Probability } \\
\text { of smoking } \\
\text { cessation }\end{array}$ & $\begin{array}{l}\text { Demographics and } \\
\text { baseline cigarette } \\
\text { dependence level }\end{array}$ & 53.3 \\
\hline $\begin{array}{l}\text { Biener et } \\
\text { al. } .^{38}(2015)\end{array}$ & USA & $\begin{array}{l}\text { Ever nicotine EC } \\
\text { users, intensive } \\
(n=111) \\
\text { intermittent } \\
(n=220)\end{array}$ & $\begin{array}{l}695 \text { smokers } \\
\text { were categorized } \\
\text { as intensive, } \\
\text { intermittent and non- } \\
\text { EC users }\end{array}$ & $\begin{array}{l}\text { Non-EC users } \\
(n=364)\end{array}$ & 2 years & $\begin{array}{l}\text { Probability } \\
\text { of smoking } \\
\text { cessation }\end{array}$ & $\begin{array}{l}\text { Demographics } \\
\text { and tobacco } \\
\text { dependence }\end{array}$ & 49.4 \\
\hline $\begin{array}{l}\text { Manzoli et } \\
\text { al. }^{39}(2015)\end{array}$ & Italy & $\begin{array}{l}\text { Ever nicotine EC } \\
\text { users }(n=223)\end{array}$ & $\begin{array}{l}1355 \text { adult (aged } \\
30-75 \text { years) smokers }\end{array}$ & $\begin{array}{l}\text { Non-EC users } \\
(n=480)\end{array}$ & 2 years & $\begin{array}{l}\text { Probability } \\
\text { of smoking } \\
\text { cessation }\end{array}$ & $\begin{array}{l}\text { Sociodemographic } \\
\text { factors alcohol } \\
\text { use and years of } \\
\text { smoking }\end{array}$ & 31.2 \\
\hline $\begin{array}{l}\text { Zhuang et } \\
\text { al. }^{40}(2016)\end{array}$ & USA & $\begin{array}{l}\text { Ever nicotine EC } \\
\text { users: long-term } \\
(n=72) \text { short- } \\
\text { term }(n=456)\end{array}$ & $\begin{array}{l}2028 \text { smokers were } \\
\text { categorized as long- } \\
\text { term and short-term } \\
\text { EC users }\end{array}$ & $\begin{array}{l}\text { Non-EC users } \\
(n=1500)\end{array}$ & 2 years & $\begin{array}{l}\text { Probability } \\
\text { of smoking } \\
\text { cessation }\end{array}$ & $\begin{array}{l}\text { Sociodemographics, } \\
\text { smoking status } \\
\text { and intention to } \\
\text { cessation }\end{array}$ & 32.6 \\
\hline $\begin{array}{l}\text { Pasquereau } \\
\text { et al. }{ }^{41} \\
(2017)\end{array}$ & France & $\begin{array}{l}\text { Ever nicotine EC } \\
\text { users }(n=252)\end{array}$ & $\begin{array}{l}2057 \text { smokers aged } \\
15-85 \text { years }\end{array}$ & $\begin{array}{l}\text { Non-EC users } \\
(n=1805) .\end{array}$ & 6 months & $\begin{array}{l}\text { Probability } \\
\text { of smoking } \\
\text { cessation }\end{array}$ & $\begin{array}{l}\text { Socioeconomic } \\
\text { variables and } \\
\text { smoking behaviors }\end{array}$ & 31.4 \\
\hline
\end{tabular}


Table 2. Continued

\begin{tabular}{|c|c|c|c|c|c|c|c|c|}
\hline $\begin{array}{l}\text { Study and } \\
\text { Year }\end{array}$ & Location & Exposure & Population & Comparison & $\begin{array}{l}\text { Follow- } \\
\qquad \text { up }\end{array}$ & Outcomes & Adjusted factors & $\begin{array}{l}\text { Lost to } \\
\text { follow-up } \\
(\%)\end{array}$ \\
\hline $\begin{array}{l}\text { Al-Delaimy } \\
\text { et al. }{ }^{42} \\
(2015)\end{array}$ & USA & $\begin{array}{l}\text { Ever nicotine EC } \\
\text { users }(n=236)\end{array}$ & 1000 adult smokers & $\begin{array}{l}\text { Non-EC users } \\
(n=177)\end{array}$ & 1 year & $\begin{array}{l}\text { Probability } \\
\text { of smoking } \\
\text { cessation }\end{array}$ & $\begin{array}{l}\text { Sociodemographic } \\
\text { characteristics }\end{array}$ & 60.0 \\
\hline $\begin{array}{l}\text { Kalkhoran et } \\
\text { al. }{ }^{43}(2019)\end{array}$ & USA & $\begin{array}{l}\text { Daily nicotine } \\
\text { EC users } \\
\text { ( } n=299) \text {, non- } \\
\text { daily EC users } \\
(n=1523)\end{array}$ & $\begin{array}{l}8218 \text { adult smokers } \\
\text { were categorized as } \\
\text { daily and non-daily } \\
\text { EC users }\end{array}$ & $\begin{array}{l}\text { Non-EC users } \\
(n=6379)\end{array}$ & 1 year & $\begin{array}{l}\text { Probability } \\
\text { of smoking } \\
\text { cessation }\end{array}$ & $\begin{array}{l}\text { Sociodemographic } \\
\text { characteristics and } \\
\text { smoking status }\end{array}$ & 2.0 \\
\hline $\begin{array}{l}\text { Jackson et } \\
\text { al. }^{44}(2019)\end{array}$ & UK & $\begin{array}{l}\text { Nicotine EC } \\
\text { users }(n=292)\end{array}$ & $\begin{array}{l}1709 \text { adult smokers } \\
\text { (aged } \geq 16 \text { years) }\end{array}$ & $\begin{array}{l}\text { Non-EC users } \\
(n=1089)\end{array}$ & 1 year & $\begin{array}{l}\text { Probability } \\
\text { of smoking } \\
\text { cessation }\end{array}$ & $\begin{array}{l}\text { Sociodemographics, } \\
\text { smoking status, } \\
\text { motivation and quit } \\
\text { attempts }\end{array}$ & 9.8 \\
\hline $\begin{array}{l}\text { Kalkhoran et } \\
\text { al. }{ }^{45}(2019)\end{array}$ & USA & $\begin{array}{l}\text { Nicotine EC } \\
\text { users }(n=762)\end{array}$ & 4948 adult smokers & $\begin{array}{l}\text { Non-EC users } \\
(n=4156)\end{array}$ & 1 year & $\begin{array}{l}\text { Probability } \\
\text { of smoking } \\
\text { cessation }\end{array}$ & $\begin{array}{l}\text { Sociodemographics, } \\
\text { smoking status, } \\
\text { education, region, } \\
\text { and nicotine } \\
\text { dependence }\end{array}$ & $<4.0$ \\
\hline $\begin{array}{l}\text { Wills et al. }{ }^{46} \\
(2016)\end{array}$ & USA & $\begin{array}{l}\text { Nicotine EC } \\
\text { users }(n=215)\end{array}$ & $\begin{array}{l}2338 \text { non-smoking } \\
\text { students (mean age } \\
14.7 \text { years) }\end{array}$ & $\begin{array}{l}\text { Non-EC users } \\
(n=926)\end{array}$ & 1 year & $\begin{array}{l}\text { Probability } \\
\text { of smoking } \\
\text { initiation }\end{array}$ & $\begin{array}{l}\text { Age, ethnicity and } \\
\text { rebelliousness }\end{array}$ & 4.20 \\
\hline $\begin{array}{l}\text { Aleyan et } \\
\text { al. }{ }^{47}(2017)\end{array}$ & Canada & $\begin{array}{l}\text { Non-susceptible } \\
\text { current nicotine } \\
\text { EC users }(n=73)\end{array}$ & $\begin{array}{l}9501 \text { grade } 9-11 \text { non- } \\
\text { smoking students }\end{array}$ & $\begin{array}{l}\text { Non- } \\
\text { susceptible } \\
\text { non-current } \\
\text { EC users } \\
(\mathrm{n}=6616)\end{array}$ & 2 years & $\begin{array}{l}\text { Probability } \\
\text { of smoking } \\
\text { initiation }\end{array}$ & $\begin{array}{l}\text { Gender, grade, } \\
\text { ethnicity and social } \\
\text { risk factors }\end{array}$ & 21.1 \\
\hline $\begin{array}{l}\text { Goldenson } \\
\text { et al. }{ }^{48} \\
(2017)\end{array}$ & USA & $\begin{array}{l}\text { Low nicotine EC } \\
\text { users }(n=52), \\
\text { medium }(n=35) \text {, } \\
\text { high users } \\
(n=21)\end{array}$ & $\begin{array}{l}3252 \text { grade } 10-11 \\
\text { non-smoking } \\
\text { students }\end{array}$ & $\begin{array}{l}\text { Placebo-EC } \\
\text { users }(n=73)\end{array}$ & 6 months & $\begin{array}{l}\text { Probability } \\
\text { of smoking } \\
\text { initiation }\end{array}$ & $\begin{array}{l}\text { Interpersonal, } \\
\text { intrapersonal } \\
\text { and demographic } \\
\text { factors }\end{array}$ & 5.0 \\
\hline $\begin{array}{l}\text { Leventhal et } \\
\text { al. }^{49}(2015)\end{array}$ & USA & $\begin{array}{l}\text { Ever nicotine EC } \\
\text { users }(n=222)\end{array}$ & $\begin{array}{l}2530 \text { non-smoking } \\
\text { teenagers (mean } \\
\text { age }=14.1 \text { years) }\end{array}$ & $\begin{array}{l}\text { Non-EC users } \\
(n=2308)\end{array}$ & $\begin{array}{l}6 \text { months } \\
12 \\
\text { months }\end{array}$ & $\begin{array}{l}\text { Probability } \\
\text { of smoking } \\
\text { initiation }\end{array}$ & $\begin{array}{l}\text { Sociodemographic, } \\
\text { environmental and } \\
\text { intrapersonal risk } \\
\text { factors }\end{array}$ & 1.1 \\
\hline $\begin{array}{l}\text { Primack et } \\
\text { al. } .^{50}(2015)\end{array}$ & USA & $\begin{array}{l}\text { Ever nicotine EC } \\
\text { users }(n=16)\end{array}$ & $\begin{array}{l}694 \text { non-smokers } \\
\text { aged } 16-26 \text { years, } \\
\text { attitudinally non- } \\
\text { susceptible to } \\
\text { smoking }\end{array}$ & $\begin{array}{l}\text { Non-EC users } \\
(n=628)\end{array}$ & 1 year & $\begin{array}{l}\text { Probability } \\
\text { of smoking } \\
\text { initiation }\end{array}$ & $\begin{array}{l}\text { Sociodemographic } \\
\text { factors and } \\
\text { maternal } \\
\text { educational level }\end{array}$ & 30.3 \\
\hline $\begin{array}{l}\text { Barrington- } \\
\text { Trimis et } \\
\text { al. }{ }^{11}(2016)\end{array}$ & USA & $\begin{array}{l}\text { Nicotine EC } \\
\text { users }(n=146)\end{array}$ & $\begin{array}{l}146 \text { non-smokers } \\
\text { (mean age } 17.4 \text { years) }\end{array}$ & $\begin{array}{l}\text { Frequency } \\
\text { matched } \\
\text { non-EC users } \\
(n=152)\end{array}$ & $\begin{array}{l}16 \\
\text { months }\end{array}$ & $\begin{array}{l}\text { Probability } \\
\text { of smoking } \\
\text { initiation }\end{array}$ & $\begin{array}{l}\text { Gender, ethnicity } \\
\text { and grade }\end{array}$ & 30.0 \\
\hline $\begin{array}{l}\text { Barrington- } \\
\text { Trimis et } \\
\text { al. }{ }^{52}(2018)\end{array}$ & USA & $\begin{array}{l}\text { Nicotine EC } \\
\text { users }(n=673)\end{array}$ & $\begin{array}{l}6258 \text { non-smoking } \\
\text { adolescents }\end{array}$ & $\begin{array}{l}\text { Non-EC users } \\
(n=3891)\end{array}$ & 2 years & $\begin{array}{l}\text { Probability } \\
\text { of smoking } \\
\text { initiation }\end{array}$ & $\begin{array}{l}\text { Gender, race/ } \\
\text { ethnicity, grade, } \\
\text { cohort }\end{array}$ & 1.8 \\
\hline $\begin{array}{l}\text { Primack et } \\
\text { al. } .^{53}(2018)\end{array}$ & USA & $\begin{array}{l}\text { Ever nicotine EC } \\
\text { users }(n=16)\end{array}$ & $\begin{array}{l}1506 \text { non-smokers } \\
\text { aged } 18-30 \text { years }\end{array}$ & $\begin{array}{l}\text { Non-EC users } \\
(n=899)\end{array}$ & $\begin{array}{l}18 \\
\text { months }\end{array}$ & $\begin{array}{l}\text { Probability } \\
\text { of smoking } \\
\text { initiation }\end{array}$ & $\begin{array}{l}\text { Sociodemographic, } \\
\text { personal, and } \\
\text { environmental } \\
\text { covariates }\end{array}$ & 39.2 \\
\hline
\end{tabular}


Table 2. Continued

\begin{tabular}{|c|c|c|c|c|c|c|c|c|}
\hline $\begin{array}{l}\text { Study and } \\
\text { Year }\end{array}$ & Location & Exposure & Population & Comparison & $\begin{array}{l}\text { Follow- } \\
\text { up }\end{array}$ & Outcomes & Adjusted factors & $\begin{array}{l}\text { Lost to } \\
\text { follow-up } \\
(\%)\end{array}$ \\
\hline $\begin{array}{l}\text { Unger et } \\
\text { al. } .^{54}(2016)\end{array}$ & USA & $\begin{array}{l}\text { Ever nicotine EC } \\
\text { users }(n=42)\end{array}$ & $\begin{array}{l}1056 \text { past } 30 \text {-day } \\
\text { non-smokers (mean } \\
\text { age } 22.7 \text { years) }\end{array}$ & $\begin{array}{l}\text { Non-EC users } \\
(n=1014)\end{array}$ & 1 year & $\begin{array}{l}\text { Probability } \\
\text { of smoking } \\
\text { initiation }\end{array}$ & $\begin{array}{l}\text { Age, sex, and } \\
\text { past-month use of } \\
\text { alcohol and other } \\
\text { tobacco products }\end{array}$ & 7.8 \\
\hline $\begin{array}{l}\text { Hammond } \\
\text { et al. }{ }^{55} \\
(2017)\end{array}$ & Canada & $\begin{array}{l}\text { Past } 30 \text {-day } \\
\text { nicotine EC } \\
\text { users }(n=487)\end{array}$ & $\begin{array}{l}17318 \text { non-smoking } \\
\text { adolescents and } \\
\text { young adults }\end{array}$ & $\begin{array}{l}\text { Non past-30 } \\
\text { EC users } \\
(n=16831)\end{array}$ & 1 year & $\begin{array}{l}\text { Probability } \\
\text { of smoking } \\
\text { initiation }\end{array}$ & $\begin{array}{l}\text { Sociodemographic } \\
\text { and smoking status }\end{array}$ & 9.5 \\
\hline $\begin{array}{l}\text { Spindle et } \\
\text { al. }{ }^{56}(2017)\end{array}$ & USA & $\begin{array}{l}\text { Ever nicotine EC } \\
\text { users }(n=153)\end{array}$ & $\begin{array}{l}\text { Non-smoking youths } \\
(\mathrm{n}=2316)\end{array}$ & $\begin{array}{l}\text { Non-EC users } \\
(n=2163)\end{array}$ & 1 year & $\begin{array}{l}\text { Probability } \\
\text { of smoking } \\
\text { initiation }\end{array}$ & $\begin{array}{l}\text { Anxiety, depression } \\
\text { peer deviance and } \\
\text { smoking status }\end{array}$ & 17.8 \\
\hline $\begin{array}{l}\text { Miech et } \\
\text { al. }{ }^{57}(2017)\end{array}$ & USA & $\begin{array}{l}\text { Ever nicotine EC } \\
\text { users }\end{array}$ & $\begin{array}{l}\text { Non-smoking youths } \\
(\mathrm{n}=246)\end{array}$ & Non-EC users & 1 year & $\begin{array}{l}\text { Probability } \\
\text { of smoking } \\
\text { initiation }\end{array}$ & $\begin{array}{l}\text { Sociodemographic } \\
\text { characteristics and } \\
\text { parental education }\end{array}$ & 57.8 \\
\hline $\begin{array}{l}\text { Chien et } \\
\text { al. }^{58}(2019)\end{array}$ & Taiwan & $\begin{array}{l}\text { Ever nicotine EC } \\
\text { users }(n=661)\end{array}$ & $\begin{array}{l}12954 \text { non-smoking } \\
\text { students }\end{array}$ & $\begin{array}{l}\text { Non-EC users } \\
(n=879)\end{array}$ & 2 years & $\begin{array}{l}\text { Probability } \\
\text { of smoking } \\
\text { initiation }\end{array}$ & $\begin{array}{l}\text { Sociodemographic } \\
\text { characteristics and } \\
\text { smoking status }\end{array}$ & 10.1 \\
\hline $\begin{array}{l}\text { Berry et al. }{ }^{59} \\
(2019)\end{array}$ & USA & $\begin{array}{l}\text { Ever nicotine EC } \\
\text { users }(n=527)\end{array}$ & $\begin{array}{l}\text { Non-smoking youths } \\
(\mathrm{n}=6123)\end{array}$ & $\begin{array}{l}\text { Non-EC users } \\
(n=5290)\end{array}$ & 2 years & $\begin{array}{l}\text { Probability } \\
\text { of smoking } \\
\text { initiation }\end{array}$ & $\begin{array}{l}\text { Sociodemographic } \\
\text { characteristics, } \\
\text { smoking status and } \\
\text { behaviors }\end{array}$ & 5.0 \\
\hline $\begin{array}{l}\text { Mcmillen et } \\
\text { al. }^{60}(2019)\end{array}$ & USA & $\begin{array}{l}\text { Ever nicotine EC } \\
\text { users }(n=195)\end{array}$ & $\begin{array}{l}\text { Non-smoking youths } \\
(n=5776)\end{array}$ & $\begin{array}{l}\text { Non-EC users } \\
(n=5473)\end{array}$ & 1 year & $\begin{array}{l}\text { Probability } \\
\text { of smoking } \\
\text { initiation }\end{array}$ & $\begin{array}{l}\text { Demographic } \\
\text { variables and } \\
\text { psychosocial } \\
\text { predictors }\end{array}$ & 1.9 \\
\hline
\end{tabular}

EC: electronic cigarette. NRT: nicotine replacement therapy.

systematic reviews ${ }^{26-30}$. Another 15 cohort studies ${ }^{46-60}$ focused on the association between e-cigarette use and subsequent smoking initiation among teenagers. Of these, six ${ }^{46,49-51,54,56}$ studies overlapped with the included systematic review ${ }^{31}$. The follow-up time varied from 6 months to 2 years.

\section{Quality assessment}

There were no $^{26}$ or one ${ }^{28}$ non-critical weakness in two systematic reviews, and these were rated as high confidence according to AMSTAR-2. Three ${ }^{29-31}$ were rated as low confidence due to the lack of a protocol and inadequate details of the included studies. One ${ }^{27}$ was of critically low confidence as it lacked a protocol and the lists of excluded studies. Details are shown in Table 3. In terms of the RCTs, the random sequence generation and allocation concealment for $3 \mathrm{RCTs}^{32-34}$ were well described and were judged as having a low risk of bias. Blinding of the participants and personnel was only reported for one $\mathrm{RCT}^{33}$ and was rated as 'low' in the blinding domain. Apart from this, the risk of performance bias was rated as 'high' in the other $4 \mathrm{RCTs}^{32,34-36}$, but the outcome assessment was blinded in these 4 RCTs and were rated as having low risk of detection bias. Intention-to-treat analysis was conducted in 5 $\mathrm{RCTs}^{32-36}$, and the attrition rate was $<10 \%$; all RCTs were assessed as having low risk of attrition bias. In terms of reporting bias, $3 \mathrm{RCTs}^{34-36}$ were assessed as 'low' since the protocols were registered and the consistency between the outcomes described in protocol and actual outcomes in the results; the remaining $2 \mathrm{RCTs}^{32,33}$ were assessed as 'unclear' due to lack of a registered protocol. The baseline data were comparable for $4 \mathrm{RCTs}^{32,34-36}$, one ${ }^{35}$ was rated as 'unclear' due to the lack of baseline data. The risk of bias summary is shown in Figure 2. The quality of 24 cohort studies scored between 5 and 7 according to the NOS grading due to the higher number of dropouts and self-reported outcomes. Overall, the quality was considered to be satisfactory. Details are shown in Table 4. 
Table 3. AMSTAR-2 assessment of the included systematic reviews

\begin{tabular}{|c|c|c|c|c|c|c|c|c|c|c|c|c|c|c|c|c|c|}
\hline \multirow[t]{2}{*}{ Study and Year } & \multicolumn{16}{|c|}{ AMSTAR-2 Items } & \multirow[t]{2}{*}{ Overall quality } \\
\hline & 1 & 2 & 3 & 4 & 5 & 6 & 7 & 8 & 9 & 10 & 11 & 12 & 13 & 14 & 15 & 16 & \\
\hline Kalkhoran et al. ${ }^{26}$ (2016) & Y & Y & Y & Y & Y & Y & Y & PY & Y & Y & $\mathrm{Y}$ & Y & Y & Y & Y & Y & high \\
\hline El Dib et al. ${ }^{27}$ (2017) & Y & $\mathrm{N}$ & $\mathrm{Y}$ & PY & Y & Y & $\mathrm{N}$ & Y & Y & Y & PY & Y & Y & $\mathrm{Y}$ & Y & Y & critically low \\
\hline Hartmann-Boyce et al. ${ }^{28}$ (2016) & Y & Y & Y & Y & Y & Y & Y & PY & Y & $\mathrm{N}$ & Y & $Y$ & Y & Y & $Y$ & Y & high \\
\hline Khoudigian et al. ${ }^{29}$ (2016) & Y & $\mathrm{N}$ & Y & Y & Y & Y & Y & PY & Y & Y & Y & Y & Y & Y & Y & Y & low \\
\hline Rahman et al. ${ }^{30}$ (2015) & Y & $\mathrm{N}$ & Y & $\mathrm{Y}$ & Y & Y & Y & $\mathrm{N}$ & Y & Y & Y & Y & Y & Y & Y & Y & low \\
\hline Soneji et al..$^{31}$ (2017) & Y & $\mathrm{N}$ & $Y$ & $\mathrm{Y}$ & Y & Y & Y & $Y$ & $\mathrm{Y}$ & $\mathrm{N}$ & Y & Y & $Y$ & Y & $Y$ & Y & low \\
\hline
\end{tabular}

Y: yes. N: no. PY: partial Y. CL: critically low. L: low. H: high. Item 1: Did the research questions and inclusion criteria for the review include the components of PICO?; Item 2: Did the report of the review contain an explicit statement that the review methods were established prior to the conduct of the review and did the report justify any significant deviations from the protocol?; Item 3: Did the review authors explain their selection of the study designs for inclusion in the review?; Item 4: Did the review authors use a comprehensive literature search strategy?; Item 5: Did the review authors perform study selection in duplicate?; Item 6: Did the review authors perform data extraction in duplicate?; Item 7: Did the review authors provide a list of excluded studies and justify the exclusions?; Item 8: Did the review authors describe the included studies in adequate detail?; Item 9: Did the review authors use a satisfactory technique for assessing the risk of bias (ROB) in individual studies that were included in the review?; Item 10: Did the review authors report on the sources of funding for the studies included in the review?; Item 11: If meta-analysis was performed, did the review authors use appropriate methods for statistical combination of results?; Item 12: If meta-analysis was performed, did the review authors assess the potential impact of ROB in individual studies on the results of the meta-analysis or other evidence synthesis?; Item 13: Did the review authors account for ROB in individual studies when interpreting/discussing the results of the review?; Item 14: Did the review authors provide a satisfactory explanation for, and discussion of, any heterogeneity observed in the result of the review?; Item 15: If they performed quantitative synthesis, did the review authors carry out an adequate investigation of publication bias (small study bias) and discuss its likely impact on the results of the review?; Item 16: Did the review authors report any potential sources of conflicts of interest, including any funding they received for conducting the review?

Figure 2. Risk of bias summary

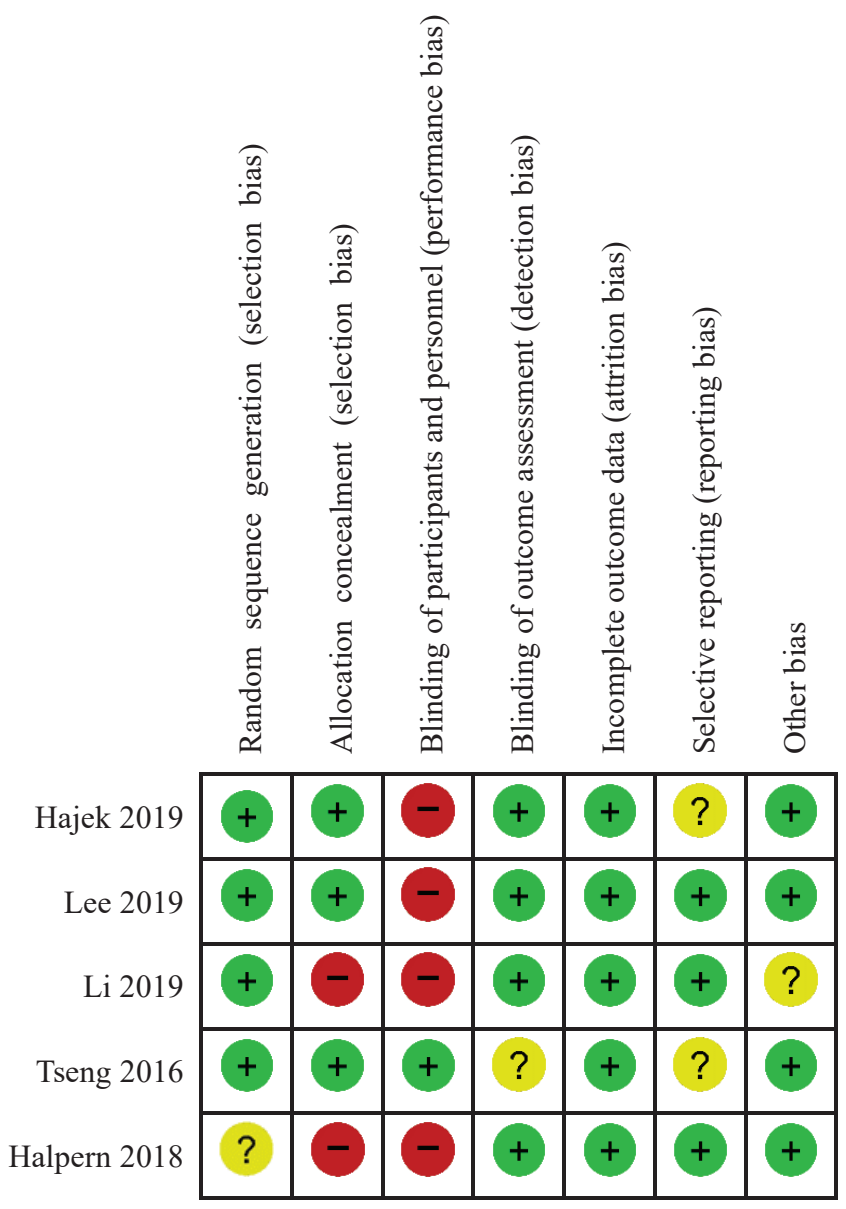

\section{Effects of interventions} Smoking cessation

Findings from 5 systematic reviews

A Cochrane systematic review ${ }^{28}$ compared e-cigarettes with placebo e-cigarettes on smoking cessation, but only $2 \mathrm{RCTs}^{5,6}$ were eligible for the meta-analysis. The abstinence rates for at least 6 months were $4 \%$ (placebo group) and 9\% (e-cigarette group), suggesting that e-cigarettes were more likely to favor cessation ( $\mathrm{RR}=2.29$; 95\% CI: $1.05-4.96$; low certainty; 2 trials, $\mathrm{n}=662$ ). This was consistent with 3 systematic reviews ${ }^{27,29,30}$. Another systematic review ${ }^{26}$ reported that the odds of cessation were $28 \%$ lower in the e-cigarette group than the non-use group ( $\mathrm{OR}=0.72$; 95\% CI: $0.57-0.91 ; 20$ trials, $\mathrm{n}=355011)$.

\section{Findings from 5 RCTs}

Five newly conducted $\mathrm{RCTs}^{32-36}$ reported the abstinence rate, with 180 of 2201 (8.2\%) smokers achieving cessation in the e-cigarette group versus 103 of $1824(5.6 \%)$ in the control group, suggesting that e-cigarettes may be superior to NRT or placebo on smoking cessation ( $\mathrm{RR}=1.55$; 95\% CI: $1.00-2.40$; $\mathrm{I}^{2}=57.6 \%$; low certainty; 5 trials, $\mathrm{n}=4025$ ) (Figure 3 , Table 5). However, the certainty of evidence was downgraded under GRADE assessment due to the small number of events $(<300)$ and the inconsistency 


\section{Review Paper}

Table 4. Newcastle-Ottawa Scale assessment of the included cohort studies

\begin{tabular}{|c|c|c|c|c|}
\hline \multirow[t]{2}{*}{ Study and Year } & \multicolumn{4}{|c|}{ Newcastle-Ottawa Scale } \\
\hline & Selection & Comparability & Outcome & Total score \\
\hline Shi et al. ${ }^{37}$ (2016) & 3 & 2 & 1 & 6 \\
\hline Biener et al. ${ }^{38}$ (2015) & 3 & 2 & 1 & 6 \\
\hline Manzoli et al. ${ }^{39}$ (2015) & 3 & 2 & 1 & 6 \\
\hline Zhuang et al. ${ }^{40}(2016)$ & 2 & 2 & 1 & 5 \\
\hline Pasquereau et al. ${ }^{41}$ (2017) & 2 & 2 & 1 & 5 \\
\hline Al-Delaimy et al. ${ }^{42}$ (2015) & 3 & 2 & 1 & 6 \\
\hline Kalkhoran et al. ${ }^{43}$ (2019) & 2 & 2 & 1 & 5 \\
\hline Jackson et al. ${ }^{44}$ (2019) & 2 & 2 & 2 & 6 \\
\hline Kalkhoran et al. ${ }^{45}$ (2019) & 2 & 2 & 1 & 5 \\
\hline Wills et al. ${ }^{46}(2016)$ & 2 & 2 & 2 & 6 \\
\hline Aleyan et al. ${ }^{47}$ (2017) & 2 & 2 & 3 & 7 \\
\hline Goldenson et al. ${ }^{48}$ (2017) & 2 & 2 & 2 & 6 \\
\hline Leventhal et al. ${ }^{49}$ (2015) & 2 & 2 & 2 & 6 \\
\hline Primack et al. ${ }^{50}$ (2015) & 3 & 2 & 1 & 6 \\
\hline Barrington-Trimis et al. ${ }^{51}$ (2016) & 2 & 2 & 1 & 5 \\
\hline Barrington-Trimis et al. ${ }^{52}$ (2018) & 1 & 2 & 2 & 5 \\
\hline Primack et al. ${ }^{53}$ (2018) & 2 & 2 & 1 & 5 \\
\hline Unger et al. ${ }^{54}(2016)$ & 2 & 2 & 2 & 6 \\
\hline Hammond et al..$^{55}$ (2017) & 2 & 2 & 2 & 6 \\
\hline Spindle et al. ${ }^{56}$ (2017) & 2 & 2 & 2 & 6 \\
\hline Miech et al. ${ }^{57}(2017)$ & 2 & 2 & 1 & 5 \\
\hline Chien et al. ${ }^{58}$ (2019) & 2 & 2 & 2 & 6 \\
\hline Berry et al..${ }^{59}$ (2019) & 2 & 2 & 1 & 5 \\
\hline Mcmillen et al. ${ }^{60}$ (2019) & 2 & 2 & 1 & 5 \\
\hline
\end{tabular}

Figure 3. Meta-analysis of RCTs comparing e-cigarettes with NRT or placebo on smoking cessation Study

ID

$\operatorname{RR}(95 \% \mathrm{Cl})$

Weight

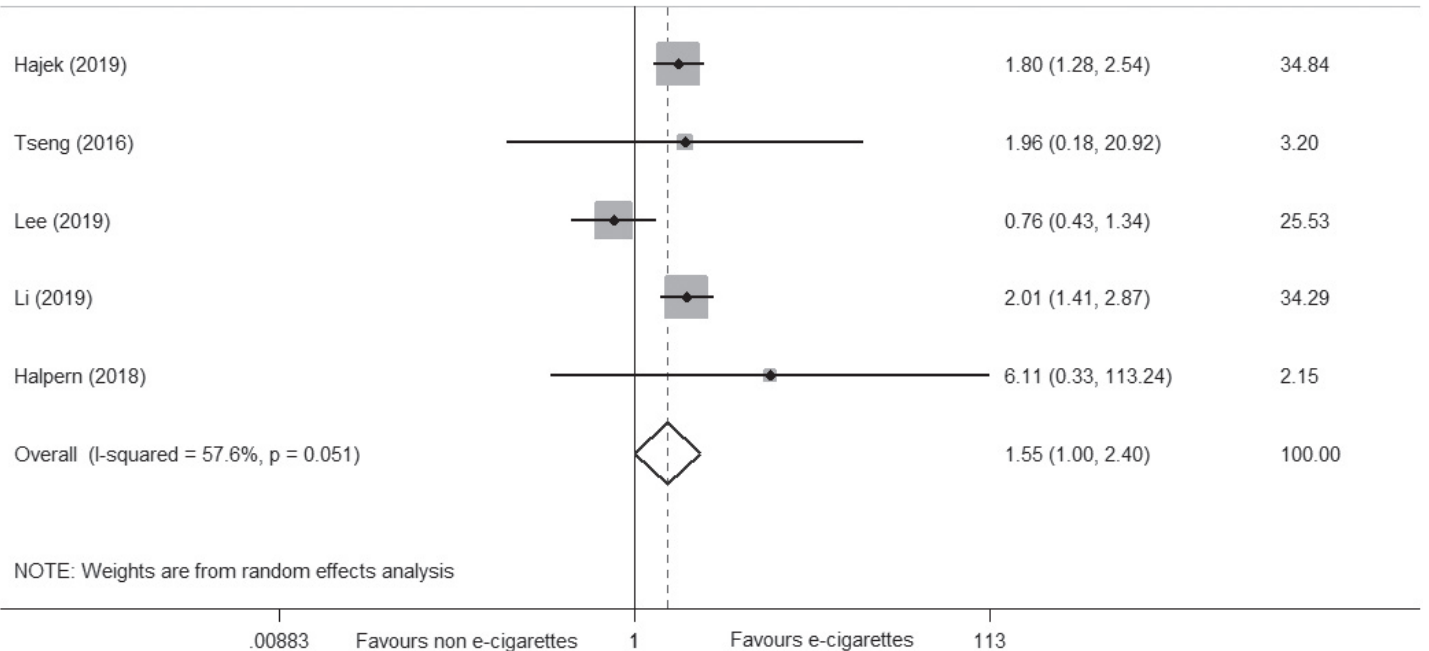


Table 5. Evidence summary of smoking cessation: e-cigarettes versus NRT or placebo

\begin{tabular}{|c|c|c|c|c|c|c|c|c|c|c|c|c|}
\hline \multicolumn{7}{|c|}{ Certainty assessment } & \multicolumn{2}{|c|}{$\begin{array}{c}\text { Number of } \\
\text { patients }\end{array}$} & \multicolumn{2}{|c|}{ Effect } & \multirow[t]{2}{*}{ Certainty } & \multirow[t]{2}{*}{ Importance } \\
\hline $\begin{array}{l}\text { No. of } \\
\text { studies }\end{array}$ & Study design & $\begin{array}{c}\text { Risk of } \\
\text { bias }\end{array}$ & Inconsistency & Indirectness & Imprecision & $\begin{array}{c}\text { Other } \\
\text { considerations }\end{array}$ & EC & $\begin{array}{l}\text { NRT or } \\
\text { placebo }\end{array}$ & $\begin{array}{l}\text { Relative } \\
(95 \% \mathrm{Cl})\end{array}$ & $\begin{array}{l}\text { Absolute } \\
(95 \% \mathrm{Cl})\end{array}$ & & \\
\hline \multicolumn{13}{|c|}{ Abstinence rate } \\
\hline 5 & $\begin{array}{l}\text { Randomized } \\
\text { trials }\end{array}$ & $\begin{array}{c}\text { Not } \\
\text { serious }\end{array}$ & Serious ${ }^{\mathrm{a}}$ & Not serious & Serious $^{b}$ & Undetected & $\begin{array}{c}180 / 2201 \\
(8.2 \%)\end{array}$ & $\begin{array}{c}103 / 1824 \\
(5.6 \%)\end{array}$ & $\begin{array}{c}\mathrm{RR}=1.55 \\
(1.00-2.40)\end{array}$ & $\begin{array}{c}31 \text { more } \\
\text { per } 1000 \\
\text { (from } 0 \\
\text { fewer to } \\
79 \text { more) }\end{array}$ & $\begin{array}{c}\oplus \oplus \bigcirc \bigcirc \\
\text { Low }\end{array}$ & Critical \\
\hline
\end{tabular}

a $\mathrm{l}^{2}=57.6 \%$. b Number of events $<300$. EC: electronic cigarette. NRT: nicotine replacement therapy. Cl: confidence interval. RR: risk ratio.

level $\left(\mathrm{I}^{2}=57.6 \%\right)$. Details are shown in Table 5 .

Findings from 9 cohort studies

Nine cohort studies ${ }^{37-45}$ reported the adjusted odds ratio (AOR) for cessation. The pooled results suggested that e-cigarettes were not associated with smoking cessation $(\mathrm{AOR}=1.16$; 95\% CI: 0.88-1.54; $\mathrm{I}^{2}=69.0 \% ; 9$ trials, $\mathrm{n}=22220$ ) (Figure 4). Subgroup analysis on the frequency of e-cigarette use suggested that intensive e-cigarette use was more effective in achieving cessation than non-use $(\mathrm{AOR}=2.03 ; 95 \%$
CI: $1.35-3.05 ; \mathrm{I}^{2}=37.8 \% ; 4$ trials, $\mathrm{n}=1144$ ) (Figure 4 ).

\section{Smoking initiation}

Findings from one systematic review

Only one systematic review ${ }^{31}$ based on cohort studies involving 17389 young people aged $14-30$ years was identified. This review indicated that ever e-cigarette users were more likely to initiate cigarette smoking at follow-up than never users $(23.2 \%$ vs $7.2 \%)$ $\left(\mathrm{AOR}=3.5\right.$; 95\% CI: $2.38-5.16 ; \mathrm{I}^{2}=56.0 \% ; 7$ trials, $\mathrm{n}=8759$ ).

Figure 4. Meta-analysis of cohort studies comparing ever e-cigarette use with non-use on smoking cessation

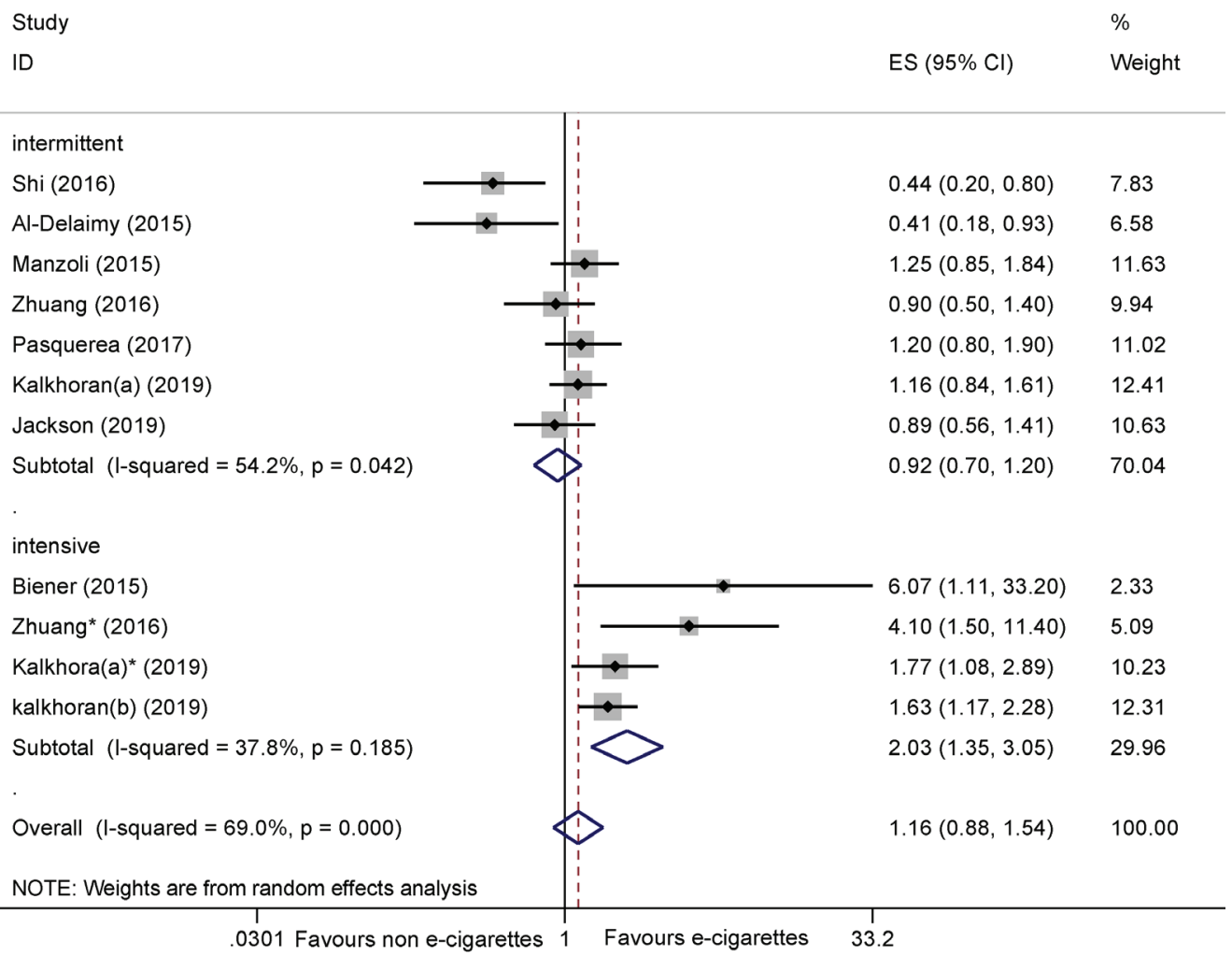




\section{Findings from 15 cohort studies}

Fifteen cohort studies ${ }^{46-60}$ were included. The pooled results suggested that ever e-cigarette users were more likely to initiate smoking than non-e-cigarette users $\left(\mathrm{AOR}=2.91 ; 95 \%\right.$ CI: $2.61-3.23 ; \mathrm{I}^{2}=61.0 \% ; 15$ trials, $n=68943$ ) (Supplementary file, Figure S1). Subgroup analysis on the frequency of e-cigarette use was not available, since only one trial ${ }^{52}$ reported the AOR of intensive and intermittent e-cigarette use. Sensitivity analysis was also unavailable due to insufficient data. The funnel plot based on the AORs of smoking initiation (Supplementary file, Figure S2) appeared to be asymmetrical, suggesting that there was potential publication bias.

\section{Adverse events}

Of the 35 included studies, adverse events were reported in 5 studies ${ }^{27-29,32,34}$. Two systematic reviews ${ }^{27,28}$ reported minor adverse short-term events related to e-cigarettes. These included irritation of the mouth and throat, cough and respiratory diseases, but long-term safety is still unknown. Two studies ${ }^{29,32}$ reported that there was no statistical difference in adverse events between the e-cigarette group and control group. One $\mathrm{RCT}^{34}$ reported that adverse events were significantly lower in the e-cigarette group (6.7\%) compared with the NRT group (17.3\%); adverse events included oral pain, cough, headache, and nausea. No serious adverse events were reported in the included trials.

\section{DISCUSSION}

\section{Main findings}

In the light of current policies on e-cigarettes in China and the US, it was timely to conduct a rapid review to comprehensively evaluate the benefits and risks of e-cigarettes. Thirty-five studies published from January 2015 to June 2020 were evaluated and included 6 systematic reviews, 24 cohort studies and 5 RCTs. The study population included adult smokers with or without intention to cease smoking and non-cigarette smoking adolescents. Four systematic reviews ${ }^{27-30}$ indicated that e-cigarettes were superior to placebo for cigarette smoking cessation. Another systematic review ${ }^{26}$ reported that the odds of cessation were $28 \%$ lower in the e-cigarette group than the nonuse group. Five RCTs (two ${ }^{33,36}$ placebo or no treatment controlled and three ${ }^{32,34,35}$ NRT controlled) reported that the abstinence rate in the e-cigarette group was $2.6 \%$ higher than that in the control group, suggesting that e-cigarettes may be more effective than NRT or placebo in achieving smoking cessation among adult smokers. However, this evidence was downgraded due to the small number of events and inconsistencies as assessed using GRADE. Nine cohort studies involving 22220 adult smokers found that e-cigarettes were not superior to non-e-cigarette use for cessation. Subgroup analysis suggested that intensive e-cigarette use (daily or regular use for at least one month) may contribute to cessation, while intermittent or irregular use did not. In light of the limited number of RCTs and the findings from cohort studies on smoking cessation, we could not draw robust conclusions from current evidence. In terms of smoking initiation, one systematic review ${ }^{31}$ reported that ever or past 30-day e-cigarette use was more likely to initiate smoking among teenagers. Updated estimates (AORs) of 15 cohort studies ${ }^{46-60}$ involving 68943 adolescents suggested that ever e-cigarette users were nearly 3 times more likely than non-users to begin smoking cigarettes. However, the evidence was limited due to potential bias (self-reported outcomes, high dropouts and variations in the length of follow-up). No serious adverse events were reported in the included studies.

\section{Comparisons with other studies}

Previous systematic reviews ${ }^{26-30}$ have merely focused on the benefits of e-cigarettes for smoking cessation or the risks for smoking initiation ${ }^{31}$. Former systematic reviews were based on primary studies such as RCTs and cohort studies. We identified the latest systematic reviews, RCTs and cohort studies published in the last six years to provide comprehensive and rapid evidence for policy makers. This is the first rapid review to evaluate the benefits and risks of e-cigarettes, comprehensively. A newly published Cochrane systematic review ${ }^{61}$ found that nicotine e-cigarettes were superior to placebo e-cigarettes or NRT for smoking cessation. This was consistent with the findings from 5 newly conducted RCTs ${ }^{32-36}$ in this rapid review. In addition, this review conducted a subgroup analysis based on the frequency of e-cigarette use.

\section{Implications}

For the study design, future studies should consider 
the frequency of e-cigarette use, the concentration of nicotine in e-cigarettes and the type of e-cigarettes, since the quitting or initiating smoking effects of e-cigarette use could be potentially affected by those factors. E-cigarette products change very rapidly, especially the Juul type e-cigarettes, which use nicotine salt and can deliver a higher dose of nicotine ${ }^{62}$. Juul is the most popular e-cigarette in the $\mathrm{US}^{63}$. Therefore, it is essential to study new products in the future in terms of their effect on quitting or initiating cigarettes. The definition of smoking cessation or smoking initiation needs to be clarified, the current standards suggest that the duration is for at least 30 days cessation or smoking initiation at follow-up. Likewise, the effects of electronic cigarettes on smoking cessation among smokers with long-term or short-term smoking may vary. Hence, different smoking durations among smokers should be well reported and stratified in future studies. In terms of outcomes, objective measurements of smoking initiation rather than self-reported measurements are warranted. Cotinine as a biomarker ${ }^{64}$ of smoke exposure can be used to predict smoking initiation among adolescents. Additionally, dropouts should be minimized as much as possible in future cohort studies. Last but not least, the long-term safety of e-cigarette use should also be a future focus. For policy makers, appropriate e-cigarette use may be potentially effective in smoking cessation for adult smokers. However, e-cigarette use in adolescents was potentially associated with subsequent smoking initiation, and therefore the sale of e-cigarettes to minors should be banned completely worldwide.

\section{Strengths and limitations}

Rapid review methodology was employed to provide timely evidence on e-cigarette use due to the urgent need to inform ongoing government policies. This is the first rapid review to evaluate the benefits and risks of e-cigarettes, comprehensively. The latest eligible systematic reviews, RCTs and cohort studies were all identified in order to provide a comprehensive evaluation on e-cigarette use. However, there are several limitations. First, similar to other rapid reviews, the search time was limited from January 2015 to June 2020, and some evidence may have been missed. The results for smoking initiation were at risk of publication bias. Second, though the evidence from
5 RCTs suggested that e-cigarettes were superior to NRT or placebo for smoking cessation, the certainty of evidence was downgraded due to imprecision and inconsistency. Additionally, evidence from 9 cohort studies suggested that ever e-cigarette use was not associated with smoking cessation. Thus, the smoking cessation effect of e-cigarettes still could not be determined based on current evidence.

\section{CONCLUSIONS}

Low certainty evidence suggests that e-cigarettes appear to be potentially effective in smoking cessation for adult smokers. However, this beneficial effect needs to be further confirmed in large sample, well designed and fully reported trials. The use of e-cigarettes in adolescents may be associated with subsequent smoking initiation. No serious adverse events were reported in the included studies, however, the long-term safety of e-cigarettes should also be a future focus.

\section{REFERENCES}

1. Kennedy RD, Awopegba A, De León E, Cohen JE. Global approaches to regulating electronic cigarettes. Tob Control. 2017;26(4):440. doi:10.1136/tobaccocontrol-2016-053179

2. Kaisar MA, Prasad S, Liles T, Cucullo L. A decade of e-cigarettes: Limited research \& unresolved safety concerns. Toxicology. 2016;365:67-75. doi:10.1016/j.tox.2016.07.020

3. Rahman MA, Hann N, Wilson A, Worrall-Carter L. Electronic cigarettes: patterns of use, health effects, use in smoking cessation and regulatory issues. Tob Induc Dis. 2014;12(December). doi:10.1186/1617-9625-12-21

4. Laverty AA, Vardavas CI, Filippidis FT. Design and marketing features influencing choice of e-cigarettes and tobacco in the EU. Eur J Public Health. 2016;26(5):838841. doi:10.1093/eurpub/ckw109

5. Bullen C, Howe C, Laugesen M, et al. Electronic cigarettes for smoking cessation: a randomized controlled trial. Lancet. 2013;382(9905):1629-1637. doi:10.1016/S0140-6736(13)61842-5

6. Caponnetto P, Campagna D, Cibella F, et al. Efficiency and Safety of an electronic cigarette (ECLAT) as tobacco cigarettes substitute: a prospective 12-month randomized control design study. Plos One. 2013;8(6):e66317. doi:10.1371/journal.pone.0066317

7. Marynak KL, Gammon DG, King BA, et al. National and state trends in sales of cigarettes and e-cigarettes, US, 2011-2015. Am J Prev Med. 2017;53(1):96-101. doi:10.1016/j.amepre.2017.01.016

8. Farsalinos KE, Poulas K, Voudris V, Le Houezec J. Electronic cigarette use in the European Union: analysis of a representative sample of 27460 Europeans from 
28 countries. Addiction. 2016;111(11):2032-2040. doi:10.1111/add.13506

9. Bauld L, MacKintosh A, Eastwood B, et al. Young People's Use of E-Cigarettes across the United Kingdom: Findings from Five Surveys 2015-2017. Int J Env Res Pub He. 2017;14(9):973. doi:10.3390/ijerph14090973

10. Singh T, Arrazola RA, Corey CG, et al. Tobacco Use Among Middle and High School Students - United States, 2011-2015. MMWR Morb Mortal Wkly Rep. 2016;65(14):361-367. doi:10.15585/mmwr.mm6514al

11. Cullen KA, Gentzke AS, Sawdey MD, et al. e-Cigarette Use Among Youth in the United States, 2019. JAMA. 2019;322(21):2095-2103. doi:10.1001/jama.2019.18387

12. National Academies of Sciences, Engineering, and Medicine, Health and Medicine Division, Board on Population Health and Public Health Practice, Committee on the Review of the Health Effects of Electronic Nicotine Delivery Systems. Public health consequences of e-cigarettes. Eaton DL, Kwan LY, Stratton K, eds. Washington, DC: National Academies Press US; 2018.

13. Miech R, Johnston L, O' Malley PM, Bachman JG, Patrick ME. Adolescent vaping and nicotine use in 2017-2018-U.S. national estimates. New Engl J Med. 2019;380(2):192-193. doi:10.1056/NEJMc1814130

14. Williams M, Bozhilov K, Ghai S, Talbot P. Elements including metals in the atomizer and aerosol of disposable electronic cigarettes and electronic hookahs. PloS One. 2017;12(4):e0175430. doi:10.1371/journal.pone.0175430

15. Gaur S, Agnihotri R. Health Effects of Trace Metals in Electronic Cigarette Aerosols-a Systematic Review. Biol Trace Elem Res. 2019;188(2):295-315. doi:10.1007/s12011-018-1423-х

16. Skotsimara G, Antonopoulos AS, Oikonomou E, et al. Cardiovascular effects of electronic cigarettes: A systematic review and meta-analysis. Eur J Prev Cardiol. 2019;26(11):1219-1228. doi:10.1177/2047487319832975

17. U.S. Food and Drug Administration. Enforcement Priorities for Electronic Nicotine Delivery System (ENDS) and Other Deemed Products on the Market Without Premarket Authorization. https://www.fda.gov/ regulatory-information/search-fda-guidance-documents/ enforcement-priorities-electronic-nicotine-deliverysystem-ends-and-other-deemed-products-market. Published April, 2020. Accessed June 5, 2020.

18. State Administration for Market Regulation. [Announcement on further protecting minors from e-cigarettes attacks]. http://gkml.samr.gov.cn/nsjg/ xyjgs/201911/t20191101_308077.html. Published November, 2019. Accessed June 5, 2020.

19. Tricco AC, Antony J, Zarin W, et al. A scoping review of rapid review methods. BMC Med. 2015;13(1):224. doi:10.1186/s12916-015-0465-6.

20. Moher D, Liberati A, Tetzlaff J, Altman DG, The PRISMA Group. Preferred Reporting Items for Systematic Reviews and Meta-Analyses: The PRISMA Statement. PLoS Med. 2009;6(7):e1000097. doi:10.1371/journal.pmed.1000097

21. Cochrane Handbook for Systematic Reviews of Interventions. Version 5.0.2. In: Higgins JPT, Green S, eds. The Cochrane Collaboration, 2008. https://training. cochrane.org/handbook/archive/v5.0.2/. Updated September, 2009. Accessed August 30, 2020.

22. Wegewitz U, Weikert B, Fishta A, Jacobs A, Pieper D. Resuming the discussion of AMSTAR: What can (should) be made better? BMC Med Res Methodol. 2016;16(1):111-118. doi:10.1186/s12874-016-0183-6

23. Wells G, Shea B, O'Connell J, et al. The Newcastle-Ottawa Scale (NOS) for assessing the quality of nonrandomised studies in meta-analyses. http://www.ohri.ca/programs/ clinical_epidemiology/oxford.asp. Accessed August 30, 2020.

24. Guyatt GH, Oxman AD, Vist GE, et al. GRADE: an emerging consensus on rating quality of evidence and strength of recommendations. BMJ. 2008;336(7650):924926. doi:10.1136/bmj.39489.470347.AD

25. Sun X, Briel M, Walter SD, Guyatt GH. Is a subgroup effect believable? Updating criteria to evaluate the credibility of subgroup analyses. BMJ. 2010. doi:10.1136/bmj.c117

26. Kalkhoran S, Glantz SA. E-cigarettes and smoking cessation in real-world and clinical settings: a systematic review and meta-analysis. Lancet Respir Med. 2016;4(2):116-128. doi:10.1016/s2213-2600(15)00521-4

27. El Dib R, Suzumura EA, Akl EA, et al. Electronic nicotine delivery systems and/or electronic non-nicotine delivery systems for tobacco smoking cessation or reduction: a systematic review and meta-analysis. BMJ Open. 2017;7(2):e012680. doi:10.1136/bmjopen-2016-012680

28. Hartmann-Boyce J, McRobbie H, Bullen C, Begh R, Stead LF, Hajek P. Electronic cigarettes for smoking cessation. Cochrane Database Syst Rev. 2016. doi:10.1002/14651858.CD010216.pub3

29. Khoudigian S, Devji T, Lytvyn L, Campbell K, Hopkins R, O'Reilly D. The efficacy and short-term effects of electronic cigarettes as a method for smoking cessation: a systematic review and a meta-analysis. Int J Public Health. 2016;61(2):257-267. doi:10.1007/s00038-016-0786-z

30. Rahman MA, Hann N, Wilson A, Mnatzaganian G, Worrall-Carter L. E-Cigarettes and Smoking Cessation: Evidence from a Systematic Review and Meta-Analysis. PloS One. 2015;10(3):e0122544. doi:10.1371/journal.pone.0122544

31. Soneji S, Barrington-Trimis JL, Wills TA, et al. Association Between Initial Use of e-Cigarettes and Subsequent Cigarette Smoking Among Adolescents and Young Adults. JAMA Pediatr. 2017;171(8):788. doi:10.1001/jamapediatrics.2017.1488

32. Hajek P, Phillips-Waller A, Przulj D, et al. A Randomized Trial of E-Cigarettes versus Nicotine-Replacement Therapy. New Engl J Med. 2019;380(7):629-637. doi:10.1056/NEJMoa1808779

33. Tseng T, Ostroff JS, Campo A, et al. A Randomized 
Trial Comparing the Effect of Nicotine Versus Placebo Electronic Cigarettes on Smoking Reduction Among Young Adult Smokers. Nicotine Tob Res. 2016;18(10):1937-1943. doi:10.1093/ntr/ntw017

34. Lee SH, Ahn SH, Cheong YS. Effect of Electronic Cigarettes on Smoking Reduction and Cessation in Korean Male Smokers: A Randomized Controlled Study. J Am Board Fam Med. 2019;32:567-574. doi:10.3122/jabfm.2019.04.180384

35. Li J, Hajek P, Pesola F, Wu Q, et al. Cost-effectiveness of e-cigarettes compared with nicotine replacement therapy in stop smoking services in England (TEC study): a randomized controlled trial. Addiction. 2020;115(3). doi:10.1111/add.14829

36. Halpern SD, Harhay MO, Saulsgiver K, Brophy C, Troxel $\mathrm{AB}$, Volpp KG. A pragmatic trial of e-cigarettes, incentives, and drugs for smoking cessation. N Engl J Med. 2018;378(24):2302-2310. doi:10.1056/NEJMsa1715757

37. Shi Y, Pierce JP, White M, et al. E-cigarette use and smoking reduction or cessation in the 2010/2011 TUS-CPS longitudinal cohort. BMC Public Health. 2016;16(1):1105. doi:10.1186/s12889-016-3770-x

38. Biener L, Hargraves JL. A Longitudinal Study of Electronic Cigarette Use Among a Population-Based Sample of Adult Smokers: Association With Smoking Cessation and Motivation to Quit. Nicotine Tob Res. 2015;17(2):127-133. doi:10.1093/ntr/ntu200

39. Manzoli L, Flacco ME, Ferrante M, et al. Cohort study of electronic cigarette use: effectiveness and safety at 24 months. Tob Control. 2017;26:284-292. doi:10.1136/tobaccocontrol-2015-052822

40. Zhuang YL, Cummins SE, Sun JY, Zhu SH. Long-term e-cigarette use and smoking cessation: a longitudinal study with US population. Tob Control. 2016;25(Suppl 1):i90-i95. doi:10.1136/tobaccocontrol-2016-053096

41. Pasquereau A, Guignard R, Andler R, Nguyen-Thanh V. Electronic cigarettes, quit attempts and smoking cessation: a 6-month follow-up. Addiction. 2017;112(9):1620-1628. doi:10.1111/add.13869

42. Al-Delaimy WK, Myers MG, Leas EC, Strong DR, Hofstetter CR. E-Cigarette Use in the Past and Quitting Behavior in the Future: A Population-Based Study. Am J Public Health. 2015;105(6):1213-1219. doi:10.2105/AJPH.2014.302482

43. Kalkhoran S, Chang Y, Rigotti NA. Electronic Cigarette Use and Cigarette Abstinence Over Two Years among U.S. Smokers in the Population Assessment of Tobacco and Health Study. Nicotine Tob Res. 2019. doi:10.1093/ntr/ntz114

44. Jackson S, Shahab L, West R, Brown J. Associations between dual use of e-cigarettes and smoking cessation: A prospective study of smokers in England. Addict Behav. 2019;103:106230. doi:10.1016/j.addbeh.2019.106230

45. Kalkhoran S, Chang Y, Rigotti N. E-cigarettes and Smoking Cessation in Smokers With Chronic Conditions. Am J Prev
Med. 2019;57:786-791. doi:10.1016/j.amepre.2019.08.017 46. Wills TA, Knight R, Sargent JD, Gibbons FX, Pagano I, Williams RJ. Longitudinal study of e-cigarette use and onset of cigarette smoking among high school students in Hawaii. Tob Control. 2016;26(1):34-39. doi:10.1136/tobaccocontrol-2015-052705

47. Aleyan S, Cole A, Qian W, Leatherdale ST. Risky business: a longitudinal study examining cigarette smoking initiation among susceptible and non-susceptible e-cigarette users in Canada. BMJ Open. 2018;8(5):e021080. doi:10.1136/bmjopen-2017-021080

48. Goldenson NI, Leventhal AM, Stone MD, McConnell RS, Barrington-Trimis JL. Associations of Electronic Cigarette Nicotine Concentration With Subsequent Cigarette Smoking and Vaping Levels in Adolescents. JAMA Pediatr. 2017;171(12):1192. doi:10.1001/jamapediatrics.2017.3209

49. Leventhal AM, Strong DR, Kirkpatrick MG, et al. Association of Electronic Cigarette Use With Initiation of Combustible Tobacco Product Smoking in Early Adolescence. JAMA. 2015;314(7):700. doi:10.1001/jama.2015.8950

50. Primack BA, Soneji S, Stoolmiller M, Fine MJ, Sargent JD. Progression to Traditional Cigarette Smoking After Electronic Cigarette Use Among US Adolescents and Young Adults. JAMA Pediatr. 2015;169(11):1018. doi:10.1001/jamapediatrics.2015.1742

51. Barrington-Trimis JL, Urman R, Berhane K, et al. E-Cigarettes and Future Cigarette Use. Pediatrics. 2016;138(1):e20160379. doi:10.1542/peds.2016-0379

52. Barrington-Trimis JL, Kong G, Leventhal AM, et al. E-cigarette Use and Subsequent Smoking Frequency Among Adolescents. Pediatrics. 2018;142(6):e20180486. doi:10.1542/peds.2018-0486

53. Primack BA, Shensa A, Sidani JE, et al. Initiation of Traditional Cigarette Smoking after Electronic Cigarette Use Among Tobacco-Naïve US Young Adults. Am J Med. 2018;131(4):443. e1-443.e9. doi:10.1016/j.amjmed.2017.11.005

54. Unger JB, Soto DW, Leventhal A. E-cigarette use and subsequent cigarette and marijuana use among Hispanic young adults. Drug Alcohol Depen. 2016;163:261-264. doi:10.1016/j.drugalcdep.2016.04.027

55. Hammond D, Reid JL, Cole AG, Leatherdale ST. Electronic cigarette use and smoking initiation among youth: a longitudinal cohort study. Can Med Assoc J. 2017;189(43):E1328-E1336. doi:10.1503/cmaj.161002

56. Spindle TR, Hiler MM, Cooke ME, Eissenberg T, Kendler KS, Dick DM. Electronic cigarette use and uptake of cigarette smoking: A longitudinal examination of U.S. college students. Addict Behav. 2017;67:66-72. doi:10.1016/j.addbeh.2016.12.009

57. Miech R, Patrick ME, O'Malley PM, Johnston LD. E-cigarette Use as a Predictor of Cigarette Smoking: Results from a One-Year Follow up of a National Sample of 12th Grade Students. Tob Control. 2017;26(E2):e106-e111. doi:10.1136/tobaccocontrol-2016-053291

58. Chien YN, Gao W, Sanna M, et al. Electronic Cigarette 
Use and Smoking Initiation in Taiwan: Evidence from the First Prospective Study in Asia. Int J Environ Res Public Health. 2019;16(7):1145. doi:10.3390/ijerph1607114

59. Berry KM , Fetterman JL, Benjamin EJ, et al. Association of Electronic Cigarette Use With Subsequent Initiation of Tobacco Cigarettes in US Youths. JAMA Netw Open. 2019;2(2). doi:10.1001/jamanetworkopen.2018.7794

60. Mcmillen RC, Klein JD, Wilson KM, Winickoff JP, Tanski S. E-Cigarette Use and Future Cigarette Initiation Among Never Smokers and Relapse Among Former Smokers in the PATH Study. Public Health Rep. 2019;134(5):528536. doi:10.1177/0033354919864369

61. Hartmann-Boyce J, McRobbie H, Lindson N, et al. Electronic cigarettes for smoking cessation. Cochrane Database Syst Rev. 2020;10:CD010216. doi:10.1002/14651858.CD010216.pub4

62. Hajek P, Pittaccio K, Pesola F, Myers Smith K, PhillipsWaller A, Przulj D. Nicotine delivery and users' reactions to Juul compared with cigarettes and other e-cigarette products. Addiction. 2020;115(6):1141-1148. doi:10.1111/add.14936

63. Huang J, Duan Z, Kwok J, et al. Vaping versus JUULing: how the extraordinary growth and marketing of JUUL transformed the US retail e-cigarette market. Tob Control. 2019;28(2):146151. doi:10.1136/tobaccocontrol-2018-054382

64. Khouja JN, Munafò MR, Relton CL, Taylor AE, Gage SH, Richmond RC. Investigating the added value of biomarkers compared with self-reported smoking in predicting future e-cigarette use: Evidence from a longitudinal UK cohort study. medRxiv. 2020. doi:10.1101/19006247
ACKNOWLEDGEMENTS

We sincerely thank Mei Han, Xun Li, Huijuan Cao, Ying Zhang and Shi-Bing Liang for providing substantive advice on the study design and revision. Many thanks to Huan Ma for his detailed revision on the format.

\section{CONFLICTS OF INTEREST}

The authors have completed and submitted the ICMJE Form for Disclosure of Potential Conflicts of Interest and none was reported.

\section{FUNDING}

This work was supported by the Key project of the National Natural Science Foundation of China (No.81830115). Nicola Robinson is funded by the Overseas Expertise Project, Ministry of Education of China (G20190001122). The funders had no role in study design, data collection and analysis, drafting the manuscript, decision to publish, or preparation of the manuscript.

\section{AUTHORS' CONTRIBUTIONS}

YYZ and JPL conceived the review and carried out the study. YYZ conducted the search, data analysis and drafted the manuscript. FLB and SJZ were involved in screening, data abstraction and quality assessment. FD and JHW provided substantive feedbacks on the study design. XWZ were involved in conducting the literature search and revision of the manuscript. JPL and NR revised the manuscript and provided constructive advice. All authors reviewed and approved the final manuscript.

PROVENANCE AND PEER REVIEW

Not commissioned; externally peer reviewed. 